\title{
Evaluation of a New Vitrification Protocol for Oocyte Cryopreservation
}

Maria Panagopoulou ${ }^{1}$, Konstantinos Charalabopoulos ${ }^{1}$, Demetrios N. Papachristou ${ }^{2}$, Elias Tsakos ${ }^{3}$ and Byron Asimakopoulos ${ }^{1 *}$

${ }^{1}$ Laboratory of Physiology, School of Medicine, Democritus University of Thrace, Greece

${ }^{2}$ Division of Endocrinology, Metropolitan Hospital, Faliron, Athens, Greece

${ }^{3}$ Gynaecology and Fertility Centre, Thessaloniki, Greece

\begin{abstract}
During the last years, vitrification was introduced into clinical practice. Although vitrification counts several years of experimental studies, this method is still under development. In this study, a new vitrification protocol was evaluated.

Materials and methods: An experimental study conducted in a University laboratory evaluated a new, short vitrification protocol including two vitrification and two thawing solutions, made in-house. The vitrification solutions contained dimethyl sulfoxide, ethylenoglycol and human serum albumin. The thawing solutions contained sucrose and human serum albumin. Rat oocytes, allocated in two groups, were used. In the control group, the oocytes were vitrified using a standard protocol. In the experimental group, the oocytes were vitrified using the new protocol. The endpoint was the survival rate of the oocytes just after thawing and 48 hours later.
\end{abstract}

Results: The survival rate just after thawing was similar in the two groups (62.5\% for the control group and $60 \%$ for the experimental group, $p=0.18$ ). However after 48 hour incubation, there was a significant difference between the two groups: $55 \%$ survival for the control group and $45 \%$ for the experimental group $(p=0.008)$.

Conclusion: The two vitrification protocols achieved similar survival rates just after thawing. However, after 48 hours incubation of thawed oocytes, the standard method had higher survival rates, than the in-house one.

\section{Keywords: Vitrification; Oocyte; DMSO; Ethylenoglycole; Sucrose}

Cryopreservation is an integral part of assisted reproduction. For years the application of slow freezing has made the cryopreservation of zygotes and embryos successful, giving to thousands of patients the opportunity to increase the effectiveness of in-vitro fertilization (IVF) cycles [1-3]. Recently, the introduction of vitrification in the everyday clinical practice entailed a higher success in the cryopreservation of embryos and mostly of oocytes [4,5]. Vitrification is considered to be more efficient method than the slow freezing. This is a quicker and simpler technique that achieves higher survival rate of embryos and oocytes [6,7]. Thus, vitrification gave a further impulse to IVF attempts.

The introduction of vitrification into IVF came along with an enhancement of the relevant research. As a result, new vitrification protocols and devices have appeared aiming to improve further the success rates of cryo cycles [8-11].

In the present study, rat oocytes were vitrified with a new vitrification protocol that was compared with another well-established and commercially available protocol. The main outcome measure was the survival rate immediately after thawing and 48 hours later.

\section{Materials and Methods}

The study was conducted in the Laboratory of Physiology, School of Medicine, Democritus University of Thrace and its design was approved by the Ethical Committee of Democritus University of Thrace.

Ten female Wistar rats (Rattus norvegicus) from the Unit of experimental animals of the Laboratory of Physiology (School of Medicine, Democritus University of Thrace) were used. The rats were housed single, in cages $20 \mathrm{~cm} \times 15 \mathrm{~cm} \times 10 \mathrm{~cm}, 12$ hours light, constant temperature and were fed ad libidum. The rats underwent ovarian stimulation with $30 \mathrm{IU}$ of human menopausal gonadotropins (Altermon, IBSA, Switzerland) i.p.; 48 hours later, they were sacrificed, the ovaries were excised and placed in pre-warmed dishes with PBS (Carl Roth, Denmark). The follicles were punctured with a needle and the cumulus-oocyte complexes were transferred into a center-well dish with Global (Lifeglobal, Canada) and incubated in humid atmosphere, $37^{\circ} \mathrm{C}$ and $5 \% \mathrm{CO}_{2}$, for one to two hours until vitrification.

The Medicult vitrification cooling and warming kit (Origio, Denmark) was used in the control group according to the instructions of the manufacturer.

In both groups, the McGill Cryoleaf (Origio, Denmark) was used as vitrification device.

In the second group, it was used an experimental vitrification protocol consisted of two solutions. The first solution (solution 1) was composed of 7.5\% dimethyl sulfoxide (DMSO) (Carl Roth, Denmark), 7.5\% ethylenoglycole (EG) (Carl Roth, Denmark), 15\% human serum albumin (Octapharma, Germany) and 70\% Ham's F10 (Sigma Aldrich, Denmark). The second solution (solution 2) was composed of $15 \%$ DMSO, 15\% EG, 15\% human serum albumin and 55\% Ham's F10. Both solutions were at room temperature. The oocytes were placed in solution 1 for three minutes and in solution 2 for 45 seconds. Then, they were quickly loaded in the vitrification device and plunged into liquid nitrogen.

*Corresponding author: Byron Asimakopoulos, PhD, Assistant Professor Laboratory of Physiology, School of Medicine, Democritus University of Thrace, 68100 Alexandroupolis, Greece, Tel: +30 2551030538; Fax: +30 2551030504 E-mail: basima@med.duth.gr

Received February 20, 2013; Accepted March 20, 2013; Published March 22 2013

Citation: Panagopoulou M, Charalabopoulos K, Papachristou DN, Tsakos E, Asimakopoulos B (2013) Evaluation of a New Vitrification Protocol for Oocyte Cryopreservation. Human Genet Embryol 3: 103. doi:10.4172/2161-0436.1000103

Copyright: (c) 2013 Panagopoulou M, et al. This is an open-access article distributed under the terms of the Creative Commons Attribution License, which permits unrestricted use, distribution, and reproduction in any medium, provided the original author and source are credited. 
The experimental thawing protocol consisted of two solutions. The first thawing solution (TS1) was composed of $1 \mathrm{M}$ sucrose (Carl Roth, Denmark), 15\% human serum albumin and 85\% Ham's F10. The second thawing solution (TS2) was composed of $0.5 \mathrm{M}$ sucrose, $15 \%$ human serum albumin and $85 \%$ Ham's F10. TS1 was placed in a centerwell dished and pre-warmed at $37^{\circ} \mathrm{C}$. TS2 was at room temperature. The device with the vitrified oocytes was immersed in TS1, the oocytes were released and remained in TS1 for one minute. Then, they were transferred into TS2 for three minutes. After this, they were washed three times in a center-well dish with PBS, transferred into a drop of Global and placed in the incubator $\left(37^{\circ} \mathrm{C}\right.$, humidified atmosphere, $\left.5 \% \mathrm{CO}_{2}\right)$.

Immediately after thawing and 48 hours later, the appearance of the oocytes was examined using an inverted microscope and a stereoscope. Survival was assessed based on the morphological integrity of oocytes.

The statistical analysis was carried out by means of chi-square test using the statistical package Statistica 6.0 (StatSoft, Tulsa, OK, USA).

\section{Results}

In each group, 40 metaphase II oocytes were vitrified. The outcome of vitrification and thawing for both groups is depicted in table 1 .

In the control group, $62.5 \%$ of oocytes survived just after thawing. During thawing, seven oocytes didn't survive in dilution 1 solution and one in dilution 2. 48 hours later, the survival rate in the control group decreased to $55 \%$.

In the experimental group, during vitrification, oocytes underwent a partial shrinkage in solution1 and a drastic reduction of their volume in solution 2 (Figure 1). During thawing, oocytes gained their

\begin{tabular}{|l|c|c|}
\hline & Control group & Experimental group \\
\hline Oocytes vitrified & 40 & 40 \\
\hline Oocytes survived just after thawing & $25(62.5 \%)^{\star}$ & $24(60 \%)^{\star}$ \\
\hline $\begin{array}{l}\text { Oocytes survived after 48 hours of } \\
\text { incubation }\end{array}$ & $22(55 \%)^{\star *}$ & $18(45 \%)^{\star *}$ \\
\hline
\end{tabular}

Table 1: Survival of rat oocytes after vitrification and thawing with the protocol of MediCult (Origio, Denmark) (control group) or the experimental protocol (experimental group). *: $p=0.18,{ }^{* *}: p=0.008$.

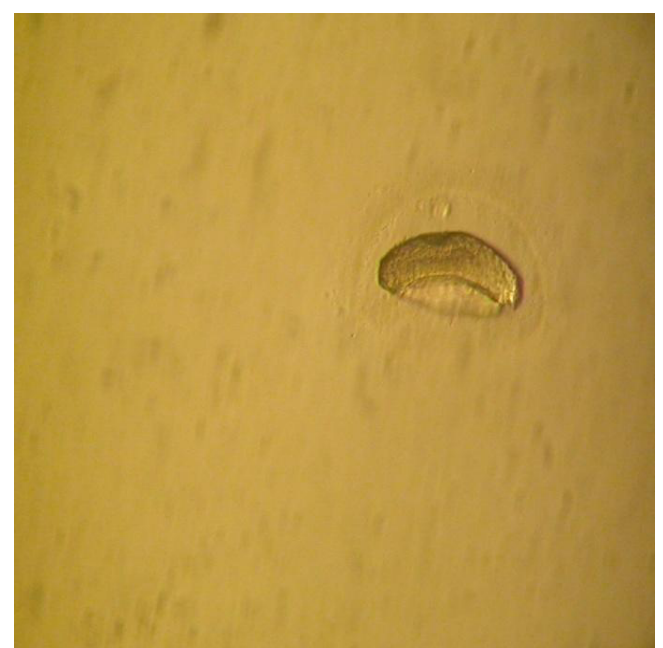

Figure 1: Rat oocyte showing full shrinkage in vitrification solution 2.

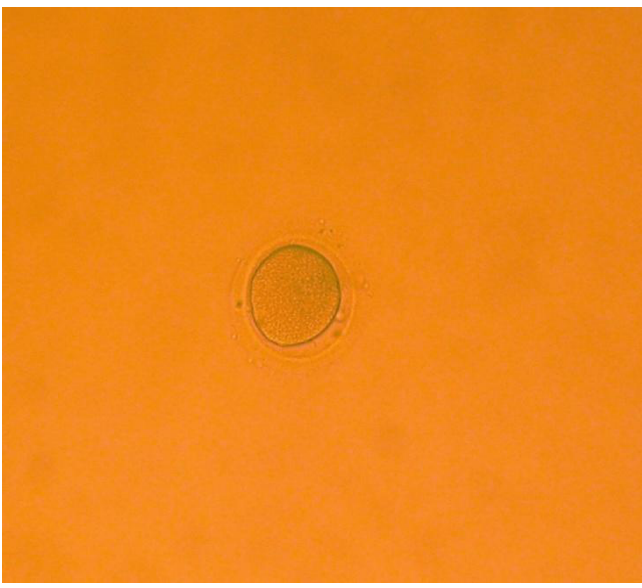

Figure 2: Rat oocyte recovered its volume in TS2.

original volume very fast in TS2 (Figure 2). Just after thawing, $60 \%$ of oocytes were alive; during thawing, in the TS1 12 oocytes and in the TS2 4 oocytes didn't survive. 48 hours later, the survival rate in the experimental group decreased to $45 \%$.

The difference between the two groups was statistically significant, only for the survival rate 48 hours after thawing ( $\mathrm{p}=0.08$ ).

\section{Discussion}

Many studies have been carried out in order to create a vitrification protocol that will be as harmless as possible for oocytes and embryos [4,5,8-12]. In this work, we designed and tested a new protocol with a short time of exposure in vitrification and thawing solutions in order to reduce the danger of toxicity.

The survival rate just after thawing was similar in the two groups $(\mathrm{p}=0.18)$. However, after 48 hours incubation, the control group had a better survival rate $(\mathrm{p}=0.008)$. The lower effectiveness of the experimental protocol was probably due to the shorter vitrification and thawing procedure, which exposed the oocytes to a higher osmotic pressure.

Namely, the vitrification and the thawing process in the experimental group were quicker than the control group, with less consecutive steps. From observations during the experiment, oocytes in the experimental group recovered faster the initial volume, while in the control group the recovery was gradual. This gradual recovery of cytoplasm, probably contributed to the higher survival rate in the control group.

\section{References}

1. Gook DA, Edgar DH (2011) Implantation rates of embryos generated from slow cooled human oocytes from young women are comparable to those of fresh and frozen embryos from the same age group. J Assist Reprod Genet 28: 11711176.

2. Edgar DH, Karani J, Gook DA (2009) Increasing dehydration of human cleavagestage embryos prior to slow cooling significantly increases cryosurvival. Reprod Biomed Online 19: 521-525.

3. Borini A, Coticchio G (2009) The efficacy and safety of human oocyte cryopreservation by slow cooling. Semin Reprod Med 27: 443-449.

4. Kim TJ, Laufer LR, Hong SW (2010) Vitrification of oocytes produces high pregnancy rates when carried out in fertile women. Fertil Steril 93: 467-474.

5. Desai N, Blackmon H, Szeptycki J, Goldfarb J (2007) Cryoloop vitrification of human day 3 cleavage-stage embryos: post-vitrification development pregnancy outcomes and live births. Reprod Biomed Online 14: 208-213. 
Citation: Panagopoulou M, Charalabopoulos K, Papachristou DN, Tsakos E, Asimakopoulos B (2013) Evaluation of a New Vitrification Protocol for Oocyte Cryopreservation. Human Genet Embryol 3: 103. doi:10.4172/2161-0436.1000103

6. Martínez-Burgos M, Herrero L, Megías D, Salvanes R, Montoya MC, et al. (2011) Vitrification versus slow freezing of oocytes: effects on morphologic appearance, meiotic spindle configuration, and DNA damage. Fertil Steril 95: 374-377.

7. Smith GD, Serafini PC, Fioravanti J, Yadid I, Coslovsky M, et al. (2010) Prospective randomized comparison of human oocyte cryopreservation with slow-rate freezing or vitrification. Fertil Steril 94: 2088-2095.

8. Sugiyama R, Nakagawa K, Shirai A, Sugiyama R, Nishi Y, et al. (2010) Clinica outcomes resulting from the transfer of vitrified human embryos using a new device for cryopreservation (plastic blade). J Assist Reprod Genet 27: 161-167.

9. Criado E, Albani E, Novara PV, Smeraldi A, Cesana A, et al. (2011) Human oocyte ultravitrification with a low concentration of cryoprotectants by ultrafast cooling: a new protocol. Fertil Steril 95: 1101-1103.

10. Kartberg AJ, Hambiliki F, Arvidsson T, Stavreus-Evers A, Svalander P (2008) Vitrification with DMSO protects embryo membrane integrity better than solutions without DMSO. Reprod Biomed Online 17: 378-384.

11. Valbuena D, Póo ME, Aguilar-Gallardo C, Martinez S, Cobo AC, et al (2012) Comparison of Cryotip vs. Cryotop for mouse and human blastomere vitrification. Fertil Steril 97: 209-217.

12. Liu WX, Lu H, Luo MJ, Xu LZ (2011) Effects of different cryoprotectants and cryopreservation protocols on the development of 2-4 cell mouse embryos. Cryo Letters 32: 240-247. 